\title{
Influência da composição de espécies florestais no microclima de sub-bosque de plantios jovens de restauração
}

\author{
Influences of tree species composition on understory \\ microclimate of young restoration plantations
}

\author{
Denise Teresinha Gonçalves Bizuti ${ }^{1}$, Ricardo Hideo Taniwaki ${ }^{1}$, Rodrigo Jesus Silva ${ }^{2}$, \\ Carolina Ortiz Rocha da Costa ${ }^{3}$, Thiago Junqueira Roncon ${ }^{1}$, \\ Ricardo Augusto Gorne Viani ${ }^{4}$ e Pedro Henrique Santin Brancalion ${ }^{5}$
}

\begin{abstract}
Resumo
Estudos que avaliem a capacidade de espécies arbóreas pioneiras em propiciar condições favoráveis à regeneração natural em plantios de restauração florestal ainda são escassos. Dessa forma, o objetivo desse estudo foi avaliar se espécies florestais pioneiras afetam de forma diferencial as condições do microclima do sub-bosque de plantios de restauração florestal. Foram selecionadas duas áreas em processo de restauração, com três e cinco anos, em região de Floresta Estacional Semidecidual em Itu/SP. Em cada área, cinco indivíduos de cinco espécies pioneiras foram escolhidos, sendo, na área de três anos, Luehea grandiflora, Schinus terebinthifolius, Solanum erianthum, Croton urucurana e Cecropia pachystachya, e na de cinco anos, L. grandiflora, S. terebinthifolius, Apeiba tibourbou, C. urucurana e S. erianthum. Cada indivíduo foi considerado uma unidade amostral. As variáveis avaliadas foram altura e cobertura florestal das espécies, umidade relativa do ar e temperatura do ar e do solo na área sob a copa dos indivíduos. Aos três anos não foram observadas diferenças nas variáveis microclimáticas de acordo com a espécie. Entretanto, aos cinco anos, as variáveis microclimáticas variaram significativamente em função da espécie. Os resultados apontam que aos três anos de idade, as espécies ainda não apresentam potencial para transformar as condições microclimáticas no sub-bosque, porém, aos cinco anos, cada espécie proporciona condições ambientais específicas. Dessa forma, ressalta-se a importância da escolha criteriosa de espécies arbóreas pioneiras como agentes de modificação ambiental e favorecimento de processos de regeneração em projetos de restauração florestal.
\end{abstract}

Palavras-chave: florestas tropicais, sucessão florestal, Mata Atlântica, monitoramento.

\begin{abstract}
Studies evaluating the pioneer tree species capacity to provide favorable conditions to natural regeneration in forest restoration plantings are still scarce. Thus, the aim of this study was to evaluate whether pioneer tree species affect differentially the microclimate conditions of the understory in forest restoration plantations. Two forest restoration sites, with three and five years old, were selected in a semi-deciduous forest in Itu city, São Paulo State, Brazil. In each site, five individuals of five pioneer species were studied. In the three years old site, Luehea grandiflora, Schinus terebinthifolius, Solanum erianthum, Croton urucurana and Cecropia pachystachya were studied, and in the five years old site, plants of the species L. grandiflora, S. terebinthifolius, Apeiba tibourbou, C. urucurana and S. erianthum were selected. Each species was considered as a sampling unit. Tree species height and canopy cover, air relative humidity and soil and air temperature were analyzed. In the three years old restoration site no effect of species in microclimatic conditions was found. However, in the five years old restoration site, microclimatic conditions were significantly different among species. Therefore, the importance of a careful choice of pioneer tree species as ecosystem engineers which modify environmental conditions and facilitate natural regeneration in forest restoration projects was emphasized.
\end{abstract}

Keywords: tropical forests, forest succession, Atlantic forest, monitoring.

\footnotetext{
${ }^{1}$ Doutorando(a) no Departamento de Ciências Florestais. USP - Universidade de São Paulo / ESALQ - Escola Superior de Agricultura "Luiz Queiroz". Av.Pádua Dias 11 - 13.418-900- Piracicaba, SP, Brasil. E-mail: denise bizuti@yahoo.com.br; rht.bio@gmail.com; thiagoroncon@hotmail.com.

2Professor Temporário do Departamento de Ciências Biológicas e da Natureza. UFAC - Universidade Federal do Acre. Distrito Industrial - 69920-900 - Rio Branco, AC, Brasil. E-mail: rodrigojsilva7@gmail.com.

${ }^{3}$ Doutoranda no Departamento de Ecologia. USP - Universidade de São Paulo / Instituto Biociências. Rua do Matão Travessa 14, 101 - 05508-090 - São Paulo, SP, Brasil. E-mail: costa cor@yahoo.com.br.

${ }^{4}$ Professor Adjunto do Departamento de Biotecnologia e Produção Vegetal e Animal. Rod. Anhanguera (SP-330), KM 174 - Caixa-postal: 153 - Araras, SP, Brasil. E-mail: viani@cca.ufscar.br.

${ }_{5}^{5}$ Professor Doutor no Departamento de Ciências Florestais. USP - Universidade de São Paulo / ESALQ - Escola Superior de Agricultura "Luiz Queiroz". Av.Pádua Dias 11 - 13.418-900- Piracicaba, SP, Brasil. E-mail: pedrob@usp.br.
}

Sci. For., Piracicaba, v. 44, n. 112, p. 971-978, dez. 2016 DOI: dx.doi.org/10.18671/scifor.v44n112.18 
Bizuti et al. - Influência da composição de espécies florestais no

microclima de sub-bosque de plantios jovens de restauração

\section{INTRODUÇÃO}

O plantio de mudas de espécies arbóreas nativas está entre as técnicas de restauração florestal mais utilizadas em regiões tropicais (LAMB et al., 2005; RODRIGUES et al., 2009), principalmente em paisagens altamente fragmentadas e com baixa resiliência (HOLL; AIDE, 2011). Entretanto, várias perguntas ainda persistem com relação ao uso e aos resultados desta técnica, como por exemplo, quais espécies plantar de modo a aumentar as chances de sucesso no processo de restauração e como cada espécie escolhida afeta o funcionamento e os serviços providos pelo ecossistema florestal formado.

Tais questões se baseiam na premissa de que existem padrões distintos de comportamento biológico entre as espécies arbóreas e que reconhecê-los contribuiria para um melhor entendimento, planejamento e manejo da restauração florestal. Com a identificação das espécies que apresentam funções naturais peculiares importantes, surge a possibilidade do uso de novos arranjos de espécies em plantios de restauração de florestas (GANDOLFI et al., 2009).

Dessa forma, o entendimento das funções ecológicas desempenhadas por cada espécie na reconstrução dos ecossistemas degradados, se torna um importante subsídio para definir a composição de espécies de plantios de recomposição florestal (GANDOLFI et al., 2009), podendo essa escolha trazer consequências benéficas para o funcionamento desses ecossistemas (SIDDIQUE et al., 2008; VIANI et al., 2015).

Cada árvore pode exercer, por meio da sua arquitetura, fenologia e características funcionais das folhas e outros órgãos, impacto relevante no funcionamento do ecossistema em restauração (OLDEMAN, 1990; JONES et al., 1997; SIDDIQUE et al., 2008; VIANI et al., 2015). Portanto, a escolha adequada de espécies arbóreas para a restauração florestal pode proporcionar o aumento da complexidade estrutural, da diversidade de habitat e da formação de uma camada de matéria orgânica sobre o solo após os primeiros anos do plantio, que frequentemente favorecem a dispersão de sementes para a área em restauração a partir das florestas do entorno, como também ajudam na supressão de gramíneas invasoras e alterações microclimáticas que favorecem o crescimento e a sobrevivência de plântulas de estágios mais tardios da sucessão florestal (ENGEL; PARROTTA, 2001).

Dessa forma, é importante reconhecer o papel que diferentes espécies arbóreas podem desempenhar para melhorar condições microclimáticas e, conjuntamente, identificar quais são capazes de minimizar a influência dos principais fatores limitantes na germinação e no estabelecimento de plântulas nos primeiros anos da trajetória sucessional, aumentando o sucesso da restauração de florestas tropicais. Assim, o objetivo desse estudo foi avaliar se espécies florestais pioneiras afetam de forma diferencial as condições de microclima do sub-bosque de plantios de restauração florestal.

\section{MATERIAL E MÉTODOS}

O estudo foi realizado no Centro de Experimentos Florestais SOS Mata Atlântica - Brasil Kirin $\left(23^{\circ} 20^{\prime} \mathrm{S}-47^{\circ} 20^{\prime} \mathrm{O}\right)$, localizado no município de Itu, na zona do médio Tietê, região sul-sudeste do estado de São Paulo, numa propriedade rural com área total de 526 ha (Figura 1). O clima predominante é caracterizado como Cwa, pela classificação de Koppen, com clima quente com verão chuvoso e inverno seco. No mês de amostragem, a temperatura mínima variou entre 16 e $25{ }^{\circ} \mathrm{C}$ e a máxima variou entre 22 e $36{ }^{\circ} \mathrm{C}$, com umidade relativa do ar instável, variando entre $43 \%$ no dia mais seco e atingindo $92 \%$ nos dias úmidos. A vegetação predominante da região é a Floresta Estacional Semidecidual, uma subformação florestal dentro do domínio fitogeográfico da Mata Atlântica, na qual parte dos indivíduos do dossel florestal perde a folha no período seco, que ocorre durante o inverno (VELOSO et al., 1991).

O local de estudo foi utilizado para a produção de café no início do século XX e, posteriormente, convertida em sua maior parte para pastagens de Urochloa decumbens para uso pecuário. Como resultado da utilização intensa do solo, a resiliência da área de estudo foi comprometida e a regeneração natural de árvores nativas é um processo incipiente ou ausente nessas áreas. Consequentemente, o reflorestamento com plantio de mudas de espécies nativas tem sido o principal método de restauração florestal empregado. 


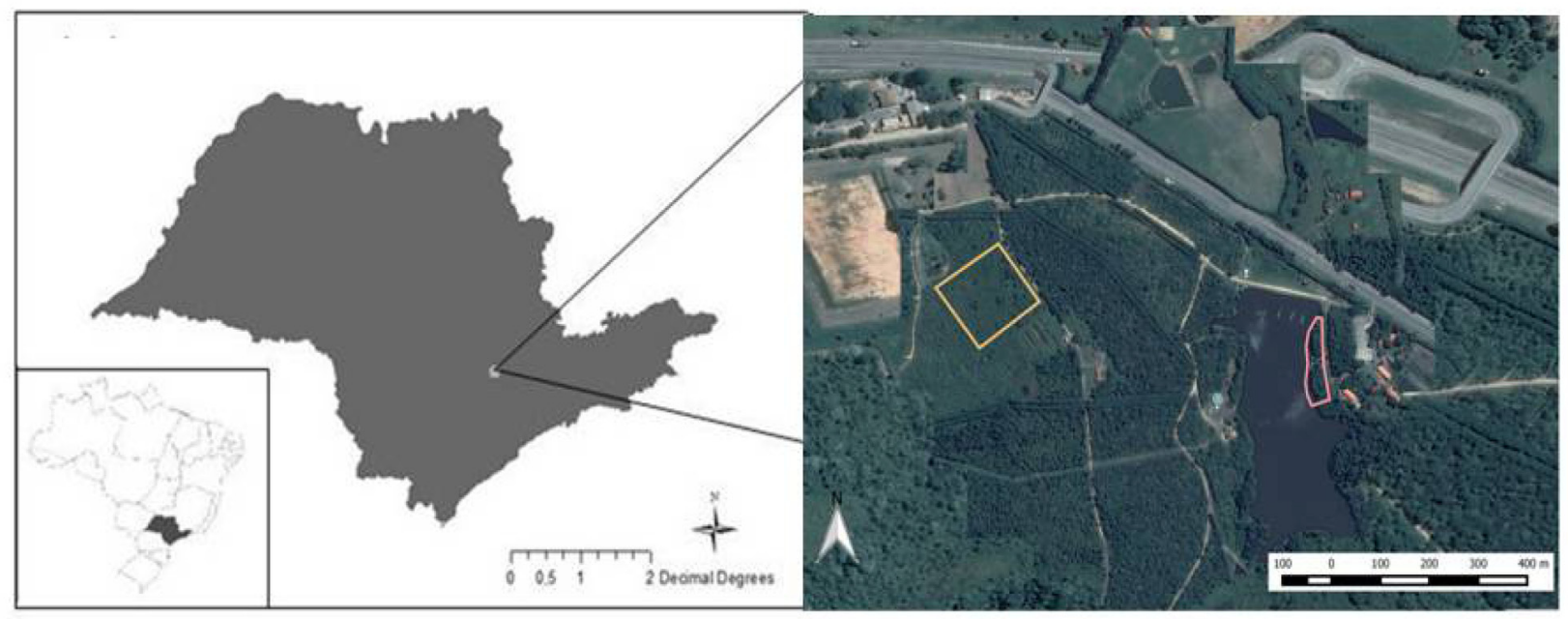

Figura 1. Localização geográfica do município de Itu-SP. Contorno em amarelo e vermelho: plantios de espécies arbóreas para fins de restauração florestal selecionados para o estudo.

Figure 1. Geographic location of the Itu municipality, São Paulo State, Brazil. Yellow and red lines: selected restoration plantations for the study.

Foram estudadas áreas em processo de restauração florestal com três e cinco anos de idade, possuindo área de 26,5 ha e 23 ha, respectivamente. As áreas foram implantadas com espaçamento de $3 \times 2 \mathrm{~m}$, com linhas alternadas de espécies de recobrimento e diversidade (RODRIGUES et al., 2009). As intervenções utilizadas para a restauração florestal nas áreas estudadas foram o controle de gramíneas invasoras com herbicida glifosato, controle de formigas com aplicação de iscas a base de sulfluramida, sulcagem e coveamento.

Nas áreas de três anos de idade foram estudadas as espécies Luehea grandiflora Mart. (Malvaceae, açoita-cavalo), Schinus terebinthifolius Raddi (Anacardiaceae, aroeira-pimenteira), Solanum erianthum D.Don (Solanaceae, fumo-bravo), Croton urucurana Baill. (Euphorbiaceae, sangra-d'água) e Cecropia pachystachya Trécul (Urticaceae, embaúba). Na área de cinco anos, foram amostradas as mesmas espécies da área anterior, com a substituição de C. pachystachya por Apeiba tibourbou Aubl. (Malvaceae, pente-de-macaco). Todas as espécies selecionadas são árvores nativas da Mata Atlântica, classificadas como espécies de recobrimento, pois apresentam rápido crescimento e uma copa ampla que recobre e sombreia o solo em poucos anos (RODRIGUES et al., 2009).

Cada espécie foi considerada uma unidade amostral e cinco representantes de cada espécie foram sistematicamente selecionadas, a partir da linha do plantio. Cada indivíduo foi identificado, numerado e a altura e a cobertura florestal pela copa foram medidas, totalizando 25 indivíduos em cada área de estudo.

As medidas das alturas totais das árvores foram obtidas com o auxílio de um hipsômetro eletrônico, que mede a altura de cada indivíduo a partir de uma relação trigonométrica. A Cobertura Florestal (CF) proporcionada pelas copas foi mensurada com o auxílio de um densiômetro convexo, que consiste em um espelho convexo com 96 pontos. Os pontos com abertura da copa (n) foram contados e submetidos à equação $\mathrm{CF}=100-\mathrm{n} .1,04$. As medidas foram aferidas em quatro direções (norte, sul, leste e oeste) e foi utilizada a média destas quatro medições como o valor de cobertura florestal da copa de cada indivíduo.

O monitoramento das variações microclimáticas foi realizado durante o período da manhã ( $9 \mathrm{~h}$ - 10:30h) e durante o período da tarde (14h - 15:30h). Em cada período foram realizadas duas campanhas amostrais. Durante as campanhas amostrais foram quantificadas três variáveis ambientais: umidade relativa do ar, temperatura do ar e do solo, sendo posteriormente utilizada a média aritmética dos dados observados para cada variável em cada período. Para obter as medidas de temperatura e umidade do ar foi utilizado um termohigrômetro (precisão de $0,5{ }^{\circ} \mathrm{C}$ e $0,1 \%$ ). A temperatura do solo foi mensurada com o auxílio de um termômetro infravermelho, sem remoção prévia da serapilheira sobre o solo. Cada variável foi aferida inicialmente na área externa ao plantio (área aberta) e, em seguida, mensuradas embaixo da copa de cada indivíduo selecionado para o estudo. A temperatura do solo foi medida em quatro direções $(\mathrm{N}, \mathrm{S}, \mathrm{L}, \mathrm{O}) \mathrm{e}$, posteriormente, foi obtida a média aritmética de temperatura do solo sob a copa de cada indivíduo. 
Para verificar as possíveis diferenças entre as características estruturais das árvores e as características microclimáticas nas áreas em restauração de três e cinco anos de idade, foi utilizada a análise de variância (ANOVA), seguida pelo teste post-hoc de Tukey para comparação de médias quando os resultados foram significativos. Para ambos foi considerado um nível de significância de 5\% $(\mathrm{p}<0,05)$. Para comparar a temperatura do ar entre as áreas de restauração e as áreas externas e para comparar a temperatura do interior das áreas em restauração de três e cinco anos de idade, foi utilizada a análise não-paramétrica de Mann-Whitney. Os pressupostos de normalidade e homogeneidade de variâncias foram verificados por meio de análise dos resíduos e testes de Shapiro-Wilk e Box-Cox, respectivamente.

\section{RESULTADOS E DISCUSSÃO}

A temperatura média do ar nas áreas externas e internas (sob as copas) no plantio foram respectivamente de 33,0 e $31,9^{\circ} \mathrm{C}$ aos três anos e de 36,4 e $33,2^{\circ} \mathrm{C}$ aos cinco anos, indicando uma redução de $1,1^{\circ} \mathrm{C}$ nos plantios de 3 anos e redução de $3,2{ }^{\circ} \mathrm{C}$ nos plantios de 5 anos sob a copa das árvores em relação à temperatura medida externamente, a pleno sol. Na área de cinco anos de idade, foi observada uma maior variação da temperatura no interior e nas áreas externas da área em restauração florestal (Figura 2).
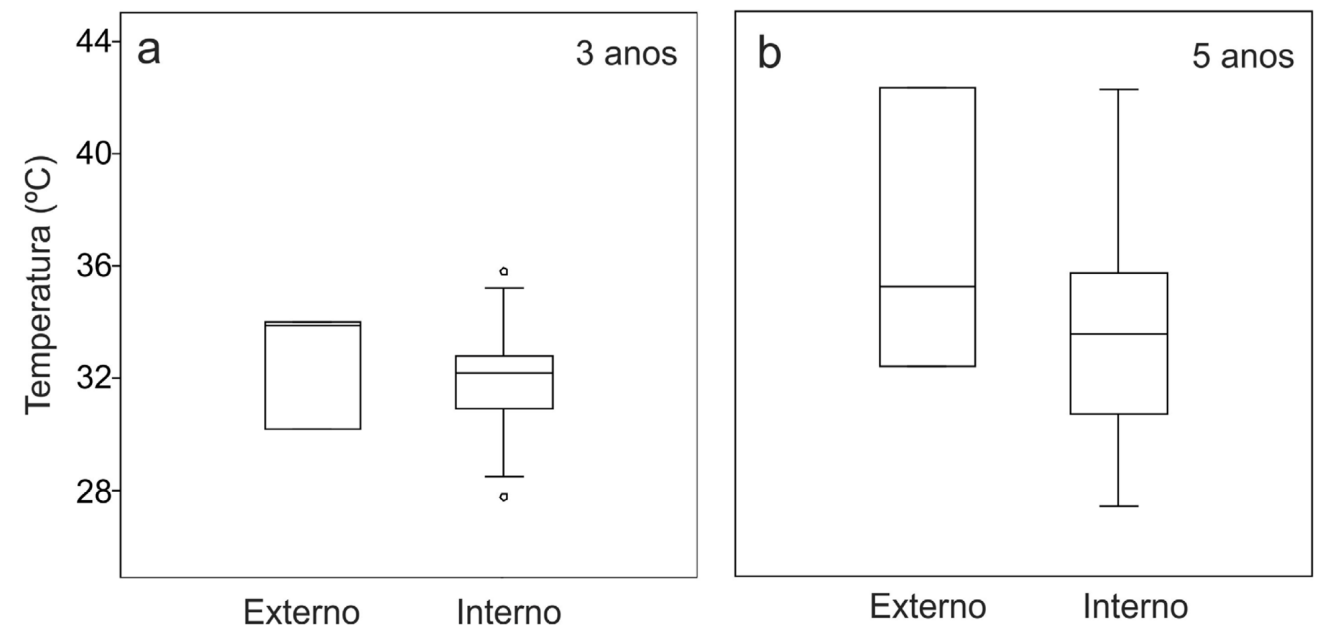

Figura 2. Temperaturas do ar externas (a pleno sol) e internas (sob a copa das árvores) em plantios de restauração florestal com três (a) e cinco (b) anos de idade. $25-75 \%$ dos quartis representados pela caixa. A mediana é representada pela linha dentro da caixa. Valores máximos e mínimos representados pelas linhas horizontais. Círculos representam valores com grande afastamento da média.

Figure 2. Internal (open area) and external (under canopies) air temperature in three (a) and five (b) years old forest restoration plantings. 25-75 percent quartiles are drawn using a box. The median is shown with a horizontal line inside the box. The minimal and maximal values are shown with short horizontal lines. Outliers are represented by circles.

Na área em processo de restauração florestal, com três anos de idade, não foram observadas diferenças significativas entre as espécies em relação às variáveis temperatura e umidade relativa do ar e temperatura do solo sob a copa (Tabela 1). A maioria das espécies não apresentou diferenças de altura, com exceção de L. grandiflora e S. terebinthifolius, que apresentaram menores alturas que as demais. Em relação à cobertura florestal, a espécie que apresentou menor porcentagem de cobertura de dossel foi $S$. terebinthifolius e a que apresentou maiores valores foi C. urucurana.

$\mathrm{Na}$ área de cinco anos foram encontradas variações microclimáticas entre as espécies (Tabela 1). A espécie S. erianthum apresentou maior temperatura do ar e do solo e menor umidade do ar. Esta espécie também apresentou a menor altura em comparação às outras. A temperatura do solo sob a copa e a altura das árvores variaram significativamente entre as espécies no plantio de cinco anos de idade. A espécie que propiciou menor temperatura do solo sob a copa foi L. grandiflora e a que apresentou maiores temperaturas do solo foi $S$. erianthum. As espécies que apresentaram maior umidade relativa do ar sob a copa foram L. grandiflora e C. urucurana. Em relação à cobertura florestal, todas as espécies apresentaram cobertura maior que 95\%. 
Tabela 1. Valores médios para as variáveis microclimáticas e estruturais avaliadas nas espécies arbóreas em plantio de restauração florestal com três e cinco anos de idade. Temperatura do solo (Ts); temperatura do ar (Tar); umidade relativa do ar, altura e cobertura florestal (CF). Médias seguidas de letras iguais na coluna não diferem entre si (teste de Tukey, $\mathrm{p}>0,05$ ).

Table 1. Mean values for microclimate and structural variables within species in three and five years old restoration plantations. Soil temperature (Ts); air temperature (Tar); relative humidity (Umidade); height (altura) and canopy cover (CF). Means followed by same letters are not significantly different (Tukey, $p>0,05)$.

\begin{tabular}{lccccc}
\hline Espécie & $\mathbf{T}$ ar $\left({ }^{\circ} \mathbf{C}\right)$ & Umidade $(\%)$ & Ts $\left({ }^{\circ} \mathbf{C}\right)$ & Altura $(\mathbf{m})$ & CF $(\%)$ \\
\hline Três anos & & & & \\
\hline C. pachystachya & $41,3 \pm 0,6 \mathrm{a}$ & $31,3 \pm 1,1 \mathrm{a}$ & $26,5 \pm 0,9 \mathrm{a}$ & $6,72 \pm 0,77 \mathrm{a}$ & 63,5 \\
C. urucurana & $40,7 \pm 0,5 \mathrm{a}$ & $31,9 \pm 1,1 \mathrm{a}$ & $25,0 \pm 0,5 \mathrm{a}$ & $4,88 \pm 1,45 \mathrm{ab}$ & 80,1 \\
L. grandiflora & $40,6 \pm 1,5 \mathrm{a}$ & $32,4 \pm 2,4 \mathrm{a}$ & $25,4 \pm 1,3 \mathrm{a}$ & $3,12 \pm 1,45 \mathrm{bc}$ & 57,8 \\
S. terebinthifolius & $41,9 \pm 0,6 \mathrm{a}$ & $32,0 \pm 1,9 \mathrm{a}$ & $26,0 \pm 0,7 \mathrm{a}$ & $2,48 \pm 0,53 \mathrm{c}$ & 56,8 \\
S. erianthum & $43,1 \pm 0,3 \mathrm{a}$ & $31,9 \pm 1,0 \mathrm{a}$ & $25,3 \pm 0,5 \mathrm{a}$ & $3,44 \pm 0,75 \mathrm{~b}$ & 76,1 \\
\hline Cinco anos & & & & & 96,3 \\
\hline A. tiborbou & $33,0 \pm 0,7 \mathrm{~b}$ & $40,3 \pm 1,8 \mathrm{ab}$ & $26,4 \pm 0,5 \mathrm{abc}$ & $8,16 \pm 1,71 \mathrm{ab}$ & 97,1 \\
C. urucurana & $32,9 \pm 0,5 \mathrm{~b}$ & $42,8 \pm 1,1 \mathrm{a}$ & $26,8 \pm 0,2 \mathrm{ab}$ & $10,02 \pm 1,61 \mathrm{a}$ & 98,4 \\
L. grandiflora & $32,5 \pm 0,5 \mathrm{~b}$ & $42,5 \pm 2,3 \mathrm{a}$ & $25,5 \pm 1,0 \mathrm{c}$ & $6,66 \pm 1,15 \mathrm{~b}$ & 96,9 \\
S. terebinthifolius & $33,0 \pm 1,0 \mathrm{~b}$ & $40,8 \pm 2,4 \mathrm{a}$ & $26,1 \pm 0,8 \mathrm{bc}$ & $7,62 \pm 2,33 \mathrm{ab}$ & 96,1 \\
S. erianthum & $34,6 \pm 0,8 \mathrm{a}$ & $36,3 \pm 2,4 \mathrm{c}$ & $27,3 \pm 0,7 \mathrm{a}$ & $4,94 \pm 0,92 \mathrm{c}$ & \\
\hline
\end{tabular}

Os resultados da comparação de variáveis ambientais, entre as espécies nas diferentes áreas, demonstram que nos primeiros três anos de um plantio de restauração florestal a identidade da espécie pouco ou não influenciou nas condições microclimáticas do sub-bosque e piso florestal. Isso porque áreas nessa idade de restauração podem ainda não apresentar indivíduos com porte suficiente para influenciar as condições microclimáticas do sub-bosque, ao passo que o dossel mais aberto favorece a maior incidência de radiação solar no solo da área, homogeneizando as características gerais do sub-bosque. Porém, já aos cinco anos, as diferentes espécies geram condições ambientais distintas sob as suas copas. É sabido que essas variáveis ambientais exercem um papel importante nos processos de transpiração, respiração, fotossíntese e, consequentemente, na produtividade vegetal (LÜTTGE, 2008; SANTOS et al., 2012; FARIAS et al., 2012).

Estudos direcionados à identificação de plantas facilitadoras e suas características são menos frequentes em ecossistemas tropicais e subtropicais devido a sua complexidade ecossistêmica. Dentre estes, a Floresta Estacional Semidecidual necessita de maiores investigações, haja vista sua peculiar dinâmica de regime luminoso, hídrico e sazonal, que comprovadamente afetam a dinâmica da regeneração natural (SOUZA et al., 2014). Ademais, um dos objetivos da restauração florestal é o de se criar futuramente e com maior brevidade possível, um ambiente favorável para a emergência e o estabelecimento de espécies vegetais regenerantes tolerantes ao sombreamento, mais tardias da sucessão florestal. Estas condições representam, dentre outras, redução da luminosidade que desfavorece a germinação de sementes de espécies ruderais do banco do solo (FIGUEIREDO et al., 2014) e temperaturas mais baixas para evitar altas taxas de respiração por essas plantas nos estágios iniciais de vida (FARIAS et al., 2012).

A redução da temperatura e o aumento da umidade do ar podem ainda favorecer o controle da infestação de gramíneas invasoras com metabolismo C4, que frequentemente limitam a restauração florestal nos trópicos (CAMPOE et al., 2010; CESAR et al., 2014). A baixa temperatura e alta umidade aumentam a sensibilidade dos estômatos para a fixação de $\mathrm{CO}_{2}$, o que beneficia a fotossíntese de espécies C3 nos estratos inferiores da estrutura florestal (JONES et al., 1997; ULRICH, 2008). Por fim, a temperatura do solo pode determinar diversos processos em áreas florestais, como disponibilidade hídrica, fluxos de $\mathrm{CO}_{2}$ (DAVIDSON et al., 1998), consumo de metano (KING; ADAMSEN, 1992) e também a mineralização de nutrientes (BONAN; VANCLEVE, 1992). Desta forma, as espécies florestais estudadas também demonstraram funções ecossistêmicas no controle da temperatura do solo, sendo este um importante indicador de processos que estão relacionados à emissão de gases de efeito estufa, merecendo maior atenção.

Diante destes aspectos, os resultados deste estudo indicam que a partir de uma dada idade da restauração florestal, as espécies arbóreas geram não só condições microclimáticas diferentes, mas muito provavelmente condições peculiares mais ou menos favoráveis para a regeneração natural e os processos de restauração florestal. Portanto, reconhecer essa capacidade nas espécies arbóreas 
utilizadas em plantios de restauração pode aumentar as chances de sucesso e/ou reduzir os custos associados com os plantios de mudas de espécies arbóreas nativas.

Ações de restauração ecológica impactam diretamente o crescimento e o desenvolvimento de mudas de árvores, todavia, é difícil reconhecer se as repostas são impulsionadas por mudanças em função das propriedades do solo, interações bióticas ou do microclima (ZAHAWI et al., 2015). Por poder criar microssítios específicos sob sua copa, cada espécie ou cada indivíduo presente no dossel das florestas tropicais e subtropicais funciona como um filtro ecológico para a comunidade regenerante, determinando parcialmente a composição, estrutura e riqueza da comunidade de plantas sob sua projeção e, através dessa permeabilidade ou impermeabilidade, afetar parcialmente a biodiversidade atual e futura da comunidade de plantas da floresta (GANDOLFI et al., 2007; ABREU et al., 2014).

Os resultados obtidos nesse trabalho levam a acreditar que existem espécies com características distintas e mais importantes para o sucesso inicial da restauração florestal, por meio do estabelecimento de condições ambientais mais favoráveis ao processo de regeneração natural. É, portanto, essencial, investigar com maior profundidade os atributos que proporcionam estas condições nas espécies arbóreas (por exemplo, densidade e perenidade da folhagem, bem como características funcionais das folhas, como área foliar específica) e como estes atributos influenciam processos ecológicos chaves à restauração de florestas, como a germinação de sementes e o estabelecimento de plântulas (BERTACCHI et al., 2012). O avanço do conhecimento neste tópico é chave para a definição de técnicas mais eficazes de implantação e manejo adaptativo da restauração nos trópicos.

De forma geral, observa-se que o conhecimento da influência de cada espécie nas alterações do microclima florestal pode ser útil tanto para restringir o número de espécies usadas em um plantio, nos casos em que se visa favorecer espécies que promovam maior sombreamento para suprimir gramíneas invasoras, como também para ampliar o número de espécies utilizado para aumentar a heterogeneidade ambiental do sub-bosque, favorecendo assim a oferta de uma maior variedade de nichos de regeneração para que um número maior de espécies possa recolonizar o sub-bosque.

\section{CONCLUSÕES}

A partir dos resultados obtidos, conclui-se que as espécies arbóreas influenciam de modo distinto o microclima do sub-bosque em plantios de restauração florestal. As influências microclimáticas variam conforme a espécie, idade e características estruturais de cada indivíduo. Dessa forma, este estudo reforça a importância da escolha minuciosa das espécies arbóreas para plantios de restauração de florestas tropicais, como também a necessidade de estudos adicionais visando selecionar espécies arbóreas que propiciem melhores condições ambientais e estímulos à regeneração natural num curto espaço de tempo.

\section{AGRADECIMENTOS}

Agradecemos a SOS Mata Atlântica, ao Roberto Cândido pelas informações cedidas, a Fundação de Amparo à Pesquisa do Estado de São Paulo FAPESP pelas bolsas cedidas a Bizuti DTG (FAPESP Processo 12/05767-5) e Taniwaki RH (FAPESP Processo 12/03527-7) e aos dois revisores anônimos que contribuíram muito para melhoria do trabalho. PHS Brancalion agradece ao CNPq por bolsa de produtividade científica (\#304817/2015-5).

\section{REFERÊNCIAS BIBLIOGRÁFICAS}

ABREU, R. C. R.; SANTOS F. F. M.; DURIGAN, G. Changes in plant community of seasonally semideciduous forest after invasion by Schizolobium parahyba at Southeastern Brazil. Acta Oecologia, Fribourg, v. 54, p. 57-64, 2014.

BERTACCHI, M. I. F.; BRANCALION, P. H. S.; BRONDANI, G. E.; MEDEIROS, J. C.; RODRIGUES, R. R. Caracterização das condições de microssítio de áreas em restauração com diferentes idades. Revista Árvore, Viçosa, v. 36, n. 5, p. 895-905, 2012. 
BONAN, G. B.; VANCLEVE, K. Soil-temperature, nitrogen mineralization, and carbon source sink relationships in boreal forests. Canadian Journal of Forest Research, Ottawa, v. 22, n. 5, p. 629-639,1992.

CAMPOE, O. C., STAPE, J. L., MENDES, J. C. T. Can intensive management accelerate the restoration of Brazil's Atlantic forests? Forest Ecology and Management, Amsterdam, v. 259, n. 9, p. 1808-1814, 2010.

CESAR, R. G.; VIANI, R. A. G.; SILVA, M. C.; BRANCALION, P. H. S. Does a native grass (Imperata brasiliensis Trin.) limit tropical forest restoration like an alien grass (Melinis minutiflora P. Beauv.)? Tropical Conservation Science, Menlo Park, v. 7, n. 4, p. 639-656, 2014.

DAVIDSON, E. A.; BELK, E.; BOONE, R. D. Soil water content and temperature as independent or confounded factors controlling soil respiration in a temperate mixed hardwood forest. Global Change Biology, Oxford, v. 4, n. 2, p. 217-227, 1998.

ENGEL, V. L.; PARROTTA, J. A. An evaluation of direct seeding for reforestation of degraded lands in central São Paulo state, Brazil. Forest Ecology and Management, Amsterdam, v. 152, n. 1-3, p. 169-191, 2001.

FARIAS, L. N.; SILVA, N. M.; DOMINGOS, S. B.; CÂNDIDO, A. K. A. A.; PIETRO-SOUZA, W. Parâmetros microclimáticos e atributos químicos e físicos do solo em área de preservação permanente situada na cabeceira do rio São Lourenço, Campo Verde - MT. Engenharia Ambiental, Espírito Santo do Pinhal, v. 9, n. 1, p. 8399, 2012.

FIGUEIREDO, P. H. A.; MIRANDA, C. C.; ARAUJO F. M.; VALCARCEL, R. Germinação ex-situ do banco de sementes do solo de capoeira em restauração florestal espontânea a partir do manejo do sombreamento. Scientia Forestalis, Piracicaba, v. 42, n. 101, p. 69-80, 2014.

GANDOLFI, S.; BELLOTTO, A.; RODRIGUES, R. R. Inserção do conceito de grupos funcionais na restauração, baseada no conhecimento da biologia das espécies. In: RODRIGUES, R. R.; BRANCALION, P. H. S.; ISERNHAGEN, I. Pacto pela restauração da mata atlântica: referencial dos conceitos e ações de restauração florestal. São Paulo: LERF/ESALQ: Instituto BioAtlântica, 2009, 256 p.

GANDOLFI, S.; JOLY, C. A.; RODRIGUES, R. R. Permeability-impermeability: canopy trees as biodiversity filters. Scientia Agricola, Piracicaba, v. 64, n. 4, p. 433-438, 2007.

HOLL, K. D.; AIDE, T. M. When and where to actively restore ecosystems? Forest Ecology and Management, Amsterdam, v. 261, n. 10, p. 1558-1563, 2011.

JONES, C. G.; LAWTON, J. H.; SHACHAK, M. Positive and negative effects of organisms as physical ecosystem engineers. Ecology, v. 78, n. 7, p. 1946-1957, 1997.

KING, G. M.; ADAMSEN, A. P. S. Effects of temperature on methane consumption in a forest soil and in pure cultures of the methanotroph methylomonas-rubra. Applied and Environmental Microbiology, Washington, v. 58, n. 9, p. 2758-2763, 1992.

LAMB, D.; ERSKINE, P. D.; PARROTTA, J. A. Restoration of degraded tropical forest landscapes. Science, Washington, v. 310, n. 5754, p.1628-1632, 2005.

LÜTTGE, U. Physiological ecology of tropical plants Berlin: Springer-Verlag, 2008. 458 p.

OLDEMAN, R. A. A. Forests: elements of silvology. Berlin: Springer-Verlag, 1990. 624 p.

RODRIGUES, R. R.; LIMA, R. A. F; GANDOLFI, S.; NAVE, A. G. On the restoration of high diversity forests: 30 years of experience in the Brazilian Atlantic Forest. Biological Conservation, Essex, v. 142, n. 6, p. 1242$1251,2009$. 
Bizuti et al. - Influência da composição de espécies florestais no

microclima de sub-bosque de plantios jovens de restauração

SANTOS, M. L. S.; FRANÇA, S.; GOMES, F. P.; NASCIMENTO, J. L.; SILVA, L. A.; MIELKE, M. S. Low light availability affects leaf gas exchange, growth and survival of Euterpe edulis seedlings transplanted into the understory of an anthropic tropical rainforest. Southern Forests: a journal of forest sciences, Grahamstown, v. 74, n. 3, p. 167-174, 2012.

SIDDIQUE, I.; ENGEL, V. L.; PARROTTA, J. A.; LAMB, D.; NARDOTO, G. B. Dominance of legume trees alters nutrient relations in mixed species forest restoration plantings within seven years. Biogeochemistry, Dordrecht, v. 88, n. 1, p. 89-101, 2008.

SOUZA F. M.; GANDOLFI, S.; RODRIGUES, R. R. Deciduousness influences the understory community in a semideciduous tropical forest. Biotropica, Lawrence, v. 46, n. 5, p. 512-515, 2014.

ULRICH, L. Physiological ecology of tropical plants. Berlin: Springer, 2008. 458 p.

VELOSO, H. P.; RANGEL FILHO, A. L. R.; LIMA, J. C. A. Classificação da vegetação brasileira, adaptada a um sistema universal. Rio de Janeiro: IBGE, 1991. 123 p.

VIANI, R. A. G., VIDAS, N. B.; PARDI, M. M.; CASTRO, D. C. V.; GUSSON, E.; BRANCALION, P. H. S. Animaldispersed pioneer trees enhance the early regeneration in Atlantic Forest restoration plantations. Natureza \& Conservação, Curitiba, v. 13, n. 1, p. 41-46, 2015.

ZAHAWI, R. A.; ECKERT, C.; CHAVES-FALLAS, J. M.; SCHWANITZ, L.; ROSALES, J. A.; HOLL, K. D. The effect of restoration treatment soils and parent tree on tropical forest tree seedling growth. Open Journal of Forestry, Delaware, v. 5, n. 2, p. 154-161, 2015.

Recebido em 16/12/2015

Aceito para publicação em 09/06/2016 